\title{
Low-Power Thermooptical Tuning of SOI Resonator Switch
}

\author{
Isa Kiyat, Student Member, IEEE, Atilla Aydinli, Member, IEEE, and Nadir Dagli, Senior Member, IEEE
}

\begin{abstract}
A wavelength selective optical switch is developed based on a high- $Q$ racetrack resonator making use of the large thermooptic coefficient of silicon. The racetrack resonator was fabricated using a silicon-on-insulator (SOI) single-mode rib waveguide. The resonator shows a high $Q$ factor of 38000 with spectral sidelobes of $11 \mathrm{~dB}$ down and can be thermooptically scanned over its full free-spectral range applying only $57 \mathrm{~mW}$ of electrical power. A low power of $17 \mathrm{~mW}$ is enough to tune the device from resonance to off-resonance state. The device functions as a wavelength selective optical switch with a 3-dB cutoff frequency of $210 \mathrm{kHz}$.
\end{abstract}

Index Terms-Integrated optics, resonator, thermooptical switch, thermooptical tuning, silicon-on-insulator (SOI) technology.

\section{INTRODUCTION}

$\mathbf{R}$ ESEARCH activities in silicon-based integrated optics have increased dramatically during the past ten years [1]. These activities in the field can be related to the development of high-quality silicon-on-insulator (SOI) wafers at low cost [1]. SOI technology opens the possibility for integration of photonic and microelectronic devices on the same wafer. Therefore, it is not surprising that a lot of the effort spent in the field of integrated optics is devoted to realization of devices on an SOI platform. So far, many integrated optical devices including light couplers, splitters, modulators, and ring resonators have been proposed and fabricated using the SOI material system [1]-[4]. Among them, ring resonators are distinguished with their high wavelength selectivity, a key issue for many device applications ranging from wavelength multiplexers [5] to modulators [6]. Recently, high- $Q$ resonator-based modulators with significantly lower switching powers have become an important alternative to Mach-Zehnder (MZ) modulators. The ability to tune the output wavelength is the first step to the development of devices for applications such as optical modulators and switches. The wavelength tuning capability is also required to fine tune to the desired wavelength when the target wavelength is not met during the fabrication process, due to imperfections or material nonuniformity. Electrooptical [6], thermooptical [7], and all-optical [8] tuning are the most commonly used mechanisms in device applications. The electrooptical effect can be implemented for fast tuning and switching on compound

Manuscript received July 18, 2005; revised November 4, 2005. This work was supported by Bilkent University under Grant Phys03-02.

I. Kiyat and A. Aydinli are with Turk Telekom Bilkent Laboratories, Department of Physics, Bilkent University, Ankara 06800, Turkey.

N. Dagli is with the Electrical and Computer Engineering Department, University of California, Santa Barbara, CA 93106 USA.

Digital Object Identifier 10.1109/LPT.2005.861951

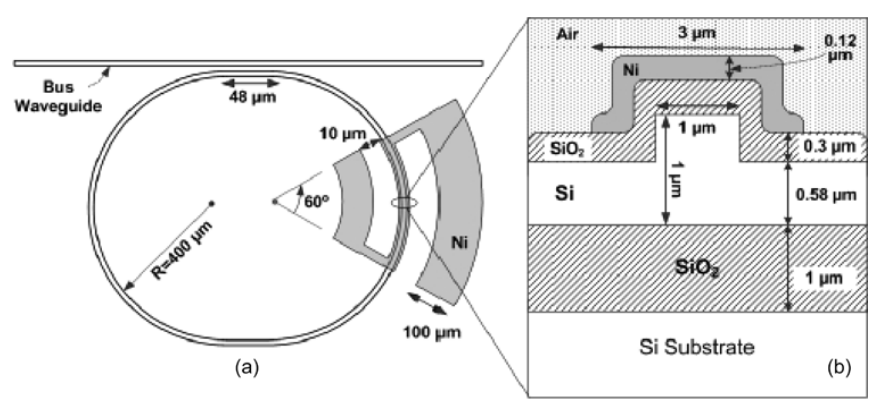

Fig. 1. Schematic views showing critical dimensions. (a) Top view of SOI resonator and (b) cross-sectional view of SOI rib waveguide.

semiconductors. However, silicon lacks inversion symmetry and an electrooptic coefficient and, therefore, does not lend itself to this mechanism. On the other hand, silicon is known for its large thermooptic coefficient [9]. Use of the thermooptical effect in silicon devices is a simple, straightforward, and cost-effective way for applications requiring tuning and moderate modulation speeds.

SOI rib waveguides having dimensions of the order of $1 \mu \mathrm{m}$ have become a new branch of optical SOI waveguides [10]. These waveguides have sizes smaller than the conventional large cross-section SOI waveguides $(3 \mu \mathrm{m})$ but larger than those of SOI nanowires $(0.4 \mu \mathrm{m})$. Their geometrically controllable birefringence has been used to realize polarization splitters [3] and polarization-independent racetrack resonators [11] on SOI. Furthermore, for a waveguide width of $1 \mu \mathrm{m}$, bending losses can be kept quite low for radii as small as $150 \mu \mathrm{m}$, thereby allowing the fabrication of high- $Q$ resonators with this dimension [12].

In this letter, we demonstrate thermooptical tuning of a high- $Q$ racetrack resonator on an SOI rib waveguide of $1-\mu \mathrm{m}$ size and its operation as a low-power optical switch. Electrical powers as low as 57 and $17 \mathrm{~mW}$ are sufficient to scan over the full free-spectral range and use the resonator as an optical switch, respectively.

\section{FABRICATION AND EXPERIMENT}

Fig. 1(a) shows a schematic top view of the designed SOI resonator with the metal heater and contact pads. The cross-sectional detail of the SOI waveguide is also shown in Fig. 1(b). This SOI waveguide geometry optimized by beam propagation (BPM) method simulations has been experimentally proved to be single mode. These low-loss single-mode rib waveguides have already been used in the demonstration of polarization splitters and high- $Q$ racetrack resonators [3], [12]. All pattern definitions throughout the fabrication of SOI resonators and their thermooptical tuning elements were done with optical 

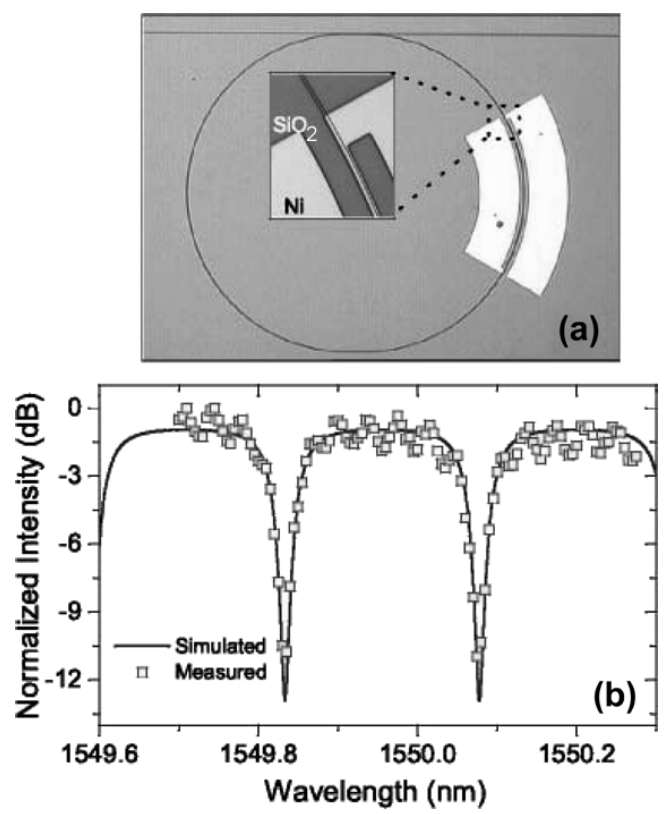

Fig. 2. (a) Optical micrographs showing top view of fabricated racetrack resonator and overlaying metal heater. (b) Measured and simulated TE transmission spectrum of fabricated SOI rib waveguide racetrack resonator.

contact photolithography. Resonator patterns were defined on an SOI wafer which had a $1.00-\mu \mathrm{m}$ top Si and a $1.00-\mu \mathrm{m}$ oxide layer on a $625-\mu \mathrm{m}$-thick substrate. The patterns were transferred to a silicon layer of $0.42 \mu \mathrm{m}$ using a reactive ion-etching (RIE) process resulting in smooth sidewalls [12]. The etch was followed by deposition of about $0.3 \mu \mathrm{m} \mathrm{SiO} \mathrm{Si}_{2}$ passivation layer using plasma-enhanced chemical vapor deposition at $250{ }^{\circ} \mathrm{C}$. The $0.12-\mu \mathrm{m}$-thick nickel heaters and contact pads were then defined through a process sequence comprising photolithography, metal sputtering, and liftoff steps. The optical micrographs of a finished resonator and its overlaying metal heater are shown in Fig. 2(a).

We cleaved the wafer to a chip of 8-mm length. Light from a high-resolution $( \pm 1 \mathrm{pm})$ external cavity tunable laser was end-fire coupled to the bus waveguide with a tapered and lensed fiber producing a $2-\mu \mathrm{m}$ spot. The light passing through the device was collected with a microscope objective and focused on a Ge detector. The measured transmission spectrum of the resonator with radius of $400 \mu \mathrm{m}$, straight coupling section of $48 \mu \mathrm{m}$, and coupling gap of $0.8 \mu \mathrm{m}$ is given in Fig. 2(b) for TE polarization. The simulated spectrum of the resonator using the well-known power transmission expression of a single-ring resonator [12, eq. 1] is also plotted in the figure. We used BPM calculated coupling factors as initial values in the simulation. After a few iterations, a coupling factor of 0.45 and resonator round trip loss of $1.57 \mathrm{~dB}$ was obtained. This is to be expected since we have already shown in [12] that SOI rib bend waveguides used in this letter with a radius of $300 \mu \mathrm{m}$ or larger do not suffer from radiation loss due to curvature. Scattering from imperfections due to fabrication and material nonuniformity are estimated to be the main sources of resonator loss. The fiber-to-fiber insertion loss was measured to be $19 \mathrm{~dB}$ with unpolished waveguide facets which were not antireflective (AR) coated. The free-spectral range can be determined to be $248 \mathrm{pm}$ from the figure.
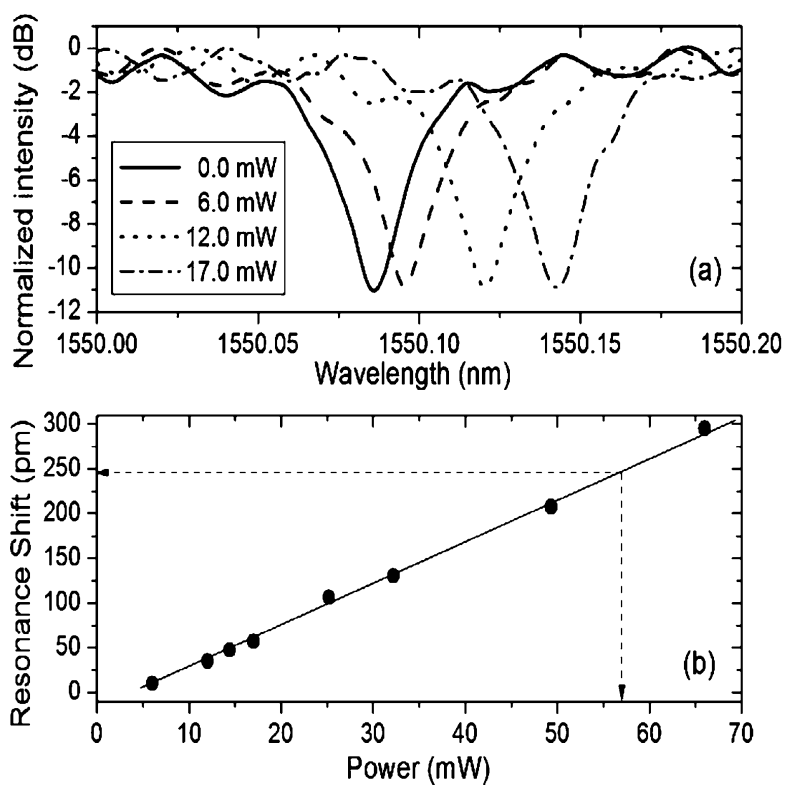

Fig. 3. (a) Measured TE transmission spectrum as electrical power applied to metal heater. (b) Shift in resonance wavelength as function of applied power.

The resonator shows good modulation characteristics with spectral side lobes of $11 \mathrm{~dB}$ down. The full-width at half-maximum (bandwidth) was extracted from the data using a curve fitting of $40 \mathrm{pm}$. Using this value with resonance wavelength and the free-spectral range of the resonator, we calculated a $Q$-factor of 38000 and a finesse value of 6.2 .

The resonance wavelengths $\left(\lambda_{0}\right)$ of a resonator are determined through the roundtrip phase term $\phi=\left(2 \pi \lambda_{0}\right) n_{\text {eff }} L=$ $m 2 \pi$ and can be tuned by either changing the resonator circumference $L$ or the effective index $n_{\text {eff }}$. Silicon is known for its large thermooptical coefficient of $1.84 \times 10^{-4}{ }^{\circ} \mathrm{C}^{-1}$, which has been employed to realize modulators on SOI wafers [9]. Thermooptical modulation of an effective refractive index is attained by placing a metal heater electrode near the waveguide. High- $Q$ resonators have narrow bandwidths and can be tuned to off-resonance with lower electrical powers [7]. After passive characterization of the resonator, we applied electrical power to the contact pads using microprobes. The heater is a $0.12-\mu \mathrm{m}$-thick and 3- $\mu \mathrm{m}$-wide nickel layer in the shape of a circular bend to assure maximum overlapping of heat flow with the optical field in the resonator. It is just above the rib of the waveguide with only a $0.3-\mu$ m-thick layer of $\mathrm{SiO}_{2}$ in between (Fig. 1). The bend-shaped heater spans an angle of $60^{\circ}$. The $100-\mu \mathrm{m}$-wide nickel contact pads were also designed in a circular shape. They are well separated from the heater [Figs. 1(a) and 2(a)]. The pads and heater combination was measured to have a resistance of $170 \Omega$. The measured transmission spectra of the resonator under applied power are shown for four different applied power values in Fig. 3(a). The transmission spectrum does not change with applied power (except for experimental error), since neither the propagation loss nor the coupling depends on the applied power within the limits used in the experiment. There is a smooth tuning of resonance wavelength with applied power. We also plot the resonance wavelength shift as a function of applied electrical power in Fig. 3(b). It can be deduced from the figure 


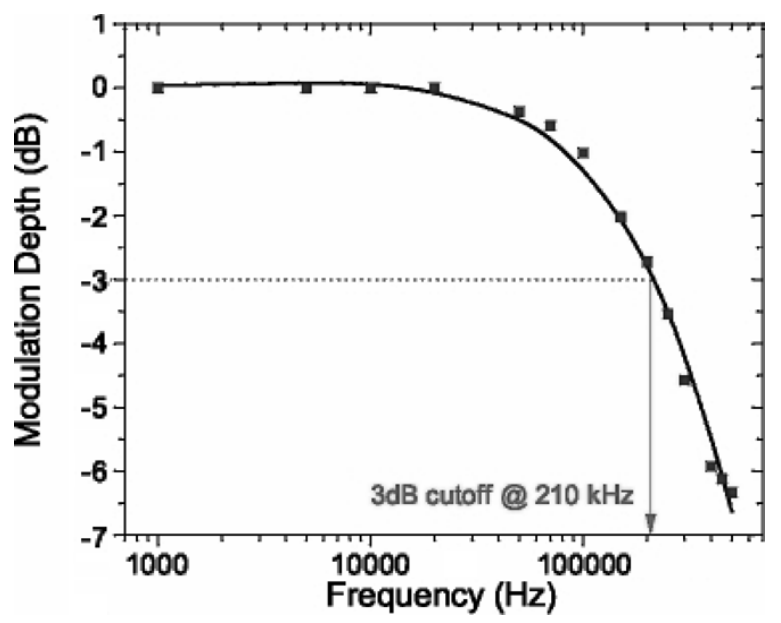

Fig. 4. Measured small signal modulation response.

that a total of $57 \mathrm{~mW}$ of electrical power is sufficient to scan the resonator over its full free-spectral range.

An equation-relating change in the effective index to the measured change in resonance wavelength can be derived from the resonance condition $\phi=m 2 \pi$ as

$$
\Delta n_{\mathrm{eff}}=\left(\frac{\Delta \lambda}{\lambda_{0}}\right) n_{\mathrm{eff}}\left(\frac{L}{L_{H}}\right)
$$

where $\Delta \lambda$ is the shift in resonance wavelength $\lambda_{0}$. The geometrical term $\left(L / L_{H}\right)$ is due to the fact that the heater does not have the same length as the circumference of the resonator. From Fig. 3(b), we find that, $17 \mathrm{~mW}$ of electrical power resulting in a shift of $57 \mathrm{pm}$ in the spectrum is sufficient to switch the resonator from the OFF (at resonance wavelength) to ON (at off-resonance wavelengths) state. Substituting $n_{\mathrm{eff}}=3.3637$ in (1), $\Delta n_{\text {eff }}=7.71 \times 10^{-4}$ is calculated for switching from the OFF to ON state at a given wavelength. We can express $\Delta n_{\text {eff }}$ as $\Delta n_{\mathrm{eff}}=\left(d n_{\mathrm{eff}} / d T\right) \Delta T \cdot d n_{\mathrm{eff}} / d T$ is a waveguide geometry-dependent term; however, we can calculate the minimum change in local temperature to be $4.19{ }^{\circ} \mathrm{C}$ by approximating $d n_{\mathrm{eff}} / d T \approx(d n / d T)_{S i}$.

To observe the frequency response of the switch, applied power was modulated by a small signal sinusoidal driving voltage. The optical signal was monitored with an InGaAs photodetector of 70-MHz bandwidth. Normalized modulation depth versus driving voltage frequency for the switch is plotted in Fig. 4. A relatively high 3-dB cutoff frequency of $210 \mathrm{kHz}$ was found for the device. This limit is due to extrinsic thermal effects since the intrinsic bandwidth limitation due to the build up and decay of the resonance is estimated to be of the order of several tens of gigahertz. To the best of our knowledge, this device is the fastest thermooptic SOI ring resonator switch, to date, with no differential control. The polarization dependence of such devices is studied in [13].

\section{CONCLUSION}

In summary, an SOI optical rib waveguide resonator is fabricated through a single-etch step and characterized to show a high $Q$ factor of 38000 . This resonator shows thermooptical tunability and operates as a wavelength-selective optical switch with low-power consumption of $17 \mathrm{~mW}$. The device was tested to function as a switch at frequencies up to $210 \mathrm{kHz}$.

\section{ACKNOWLEDGMENT}

The authors would like to thank Dr. A. Dane and A. Kocabas for their help with the frequency response measurements and useful discussions.

\section{REFERENCES}

[1] B. Jalali, S. Yagnanarayanan, T. Yoon, T. Yoshimoto, I. Rendina, and F. Coppinger, "Advances in silicon-on-insulator optoelectronics," IEEE J. Sel. Topics Quantum Electron., vol. 4, no. 6, pp. 938-947, Dec. 1998.

[2] P. D. Trinh, S. Yagnanarayanan, and B. Jalali, "Integrated optical directional couplers in silicon-on-insulator," Electron. Lett., vol. 31, no. 24, pp. 2097-2098, 1995.

[3] I. Kiyat, A. Aydinli, and N. Dagli, "A compact silicon-on-insulator polarization splitter," IEEE Photon. Technol. Lett., vol. 17, no. 1, pp. 100-102, Jan. 2005

[4] B. E. Little, J. S. Foresi, G. Steinmeyer, E. R. Thoen, S. T. Chu, H. Haus, E. P. Ippen, L. C. Kimerling, and W. Greene, "Ultra-compact $\mathrm{Si}_{-} \mathrm{SiO}_{2}$ microring resonator optical channel dropping filters," IEEE Photon. Technol. Lett., vol. 10, no. 4, pp. 549-551, Apr. 1998.

[5] E. Little, S. T. Chu, P. P. Absil, J. V. Hryniewicz, F. G. Johnson, F. Seifert, D. Gill, V. Van, O. King, and M. Trakalo, "Very high-order microring resonator filters for WDM applications," IEEE Photon. Technol. Lett., vol. 16, no. 10, pp. 2263-2265, Oct. 2004.

[6] T. Sadagopan, S. June Choi, K. Djordjev, and P. D. Dapkus, "Carrierinduced refractive index changes in InP-based circular microresonators for low-voltage high-speed modulation," IEEE Photon. Technol. Lett., vol. 17, no. 2, pp. 414-416, Feb. 2005.

[7] W. M. J. Green, R. K. Lee, G. A. DeRose, A. Scherer, and A. Yariv, "Hybrid InGaAsP-InP Mach-Zehnder racetrack rasonator resonator for thermooptic switching and coupling control," Opt. Expr, vol. 13, no. 5, pp. 1651-1659, 2005

[8] V. R. Almeida, C. A. Barrios, R. R. Panepucci, M. Lipson, M. A. Foster, D. G. Ouzounov, and A. L. Geata, "All-optical switching on a silicon chip," Opt. Lett., vol. 29, no. 24, pp. 2867-2869, 2004.

[9] R. L. Espinola, M. C. Tsai, J. T. Yardley, and R. M. Osgood Jr., "Fast and low-power thermooptic switch on thin silicon-on-insulator," IEEE Photon. Technol. Lett., vol. 15, no. 10, pp. 1366-1368, Oct. 2003.

[10] W. S. P. Chan, C. E. Png, S. T. Lim, G. T. Reed, and V. M. N. Passaro, "Single-mode and polarization-independent silicon-on-insulator waveguides with small cross section," J. Lightw. Technol., vol. 23, no. 6, pp. 2103-2111, Jun. 2005.

[11] W. R. Headley, G. T. Reed, S. Howe, A. Liu, and M. Paniccia, "Polarization-independent optical racetrack resonators using rib waveguides on silicon-on-insulator," Appl. Phys. Lett., vol. 85, no. 23, pp. 5523-5525, 2004.

[12] I. Kiyat, A. Aydinli, and N. Dagli, "High-Q silicon-on-insulator optical rib waveguide racetrack resonators," Opt. Expr., vol. 13, no. 6, pp. 1900-1905, 2005

[13] — , "Compact large cross section silicon-on-insulator racetrack resonators," IEEE Photon. Technol. Lett., vol. 17, no. 10, pp. 2098-2100, Oct. 2005 . 